\title{
Noise and Full Counting Statistics of Incoherent Multiple Andreev Reflection
}

\author{
S. Pilgram and P. Samuelsson \\ Département de Physique Théorique, Universitè de Genéve, CH-1211, Genève 4, Switzerland
}

(Dated: November 9, 2018)

\begin{abstract}
We present a general theory for the full counting statistics of multiple Andreev reflections in incoherent superconducting-normal-superconducting contacts. The theory, based on a stochastic path integral approach, is applied to a superconductor-double barrier system. It is found that all cumulants of the current show a pronounced subharmonic gap structure at voltages $V=2 \Delta / e n$. For low voltages $V \ll \Delta / e$, the counting statistics results from diffusion of multiple charges in energy space, giving the $p$ th cumulant $\left\langle Q^{p}\right\rangle \propto V^{2-p}$, diverging for $p \geq 3$. We show that this low-voltage result holds for a large class of incoherent superconducting-normal-superconducting contacts.
\end{abstract}

PACS numbers: 73.23.-b, 05.40.-a, 72.70.+m, 02.50.-r, 76.36.Kv

Electrical transport in superconducting contacts is a subject of persistent interest. The basic mechanism of transport in voltage biased contacts, Multiple Andreev Reflections (MAR), was described by Klapwijk, Blonder and Tinkham [1] twenty years ago. Since then, a large number of works have investigated the current-voltage characteristics in superconducting contacts. As a prominent example, experiments on superconducting atomic point contacts have been explained remarkably well by coherent MAR-theories [2].

Recently, interest has turned to the properties of current noise. Shot noise was measured in several types of superconducting contacts 3, 4, 5 and was theoretically studied in both the coherent [6, 7] and incoherent limit [8, 9, 10]. Very recently, the full distribution of current fluctuations, the Full Counting Statistics (FCS) 11] of MAR in coherent superconducting contacts was calculated [12, 13]. The FCS revealed explicitly that charge in coherent superconducting-normalsuperconducting (SNS) systems is transfered in multiples of the elementary charge. In diffusive SNS-systems, this leads to a characteristic power-law divergence of the shot noise at low voltages, $S_{I} \sim V^{-1 / 2}$ [7]. However, in many experiments quantum coherence is suppressed [5]. Interestingly, calculations for incoherent diffusive structures 8, 9, 10] show that the low-voltage shot noise is wellbehaved and can be explained in terms of an effective electron temperature of the order of the superconducting gap $\Delta$. This naturally raises the question about the role of multiple charge transfer in incoherent SNS-systems.

We address this question by developing a theory for the FCS of incoherent MAR in semiclassical SNS-systems, based on a stochastic path integral [14, 15] approach. As an illustrative example, we investigate the superconducting double-barrier model of Octavio et al. (OTBK) [16], focusing on the voltage dependence of the FCS. For a generic incoherent SNS-system, we show that for low voltage, $e V \ll \Delta$, the generating function is

$$
S(\chi)=\frac{\tau e V^{2} G}{2 \Delta} \operatorname{asinh}^{2} \sqrt{\exp \left(\chi^{2 \Delta / e V)-1}\right.}
$$

with $\tau$ the measurement time, $\chi$ the counting field and $G$ the linear, low-voltage conductance. This result, an effect of diffusive motion of the quasiparticles in energy space, is just the FCS for a metallic diffusive wire [15, 17] with an effective voltage dependent charge $e \rightarrow e(2 \Delta / e V)$. Unlike coherent diffusive systems [7], the cumulants $\left\langle Q^{p}\right\rangle=$ $\partial^{p} S /\left.\partial \chi^{p}\right|_{\chi=0} \sim V^{2-p}$ diverge only for $p \geq 3$. The successful measurement of the third cumulant $\left\langle Q^{3}\right\rangle$ in normal conducting tunnel junctions 18 shows that an experimental test of our prediction is feasible.

The model - The studied geometry is shown in Fig. [1 Two superconducting electrodes (S) with gaps $\Delta$ are connected via a ballistic normal conducting region $(\mathrm{N})$ with $M$ transport modes. The NS-interfaces, with equal mode-independent normal state transparencies $\Gamma$, are characterized by energy dependent probabilities 19] for normal/Andreev reflection and transmission $R_{N / A}(E), T_{N / A}(E)$. A voltage $V$ is applied between the two superconductors. For $e V<2 \Delta$, transport of quasiparticles across the gap takes place via MAR along the energy ladder shown in Fig. 1]

In contrast to previous works on FCS of MAR 12, 13, we will consider the incoherent limit, i.e. with suppressed proximity and dc/ac-Josephson effect. This experimentally interesting limit [5] is relevant for strong dephasing

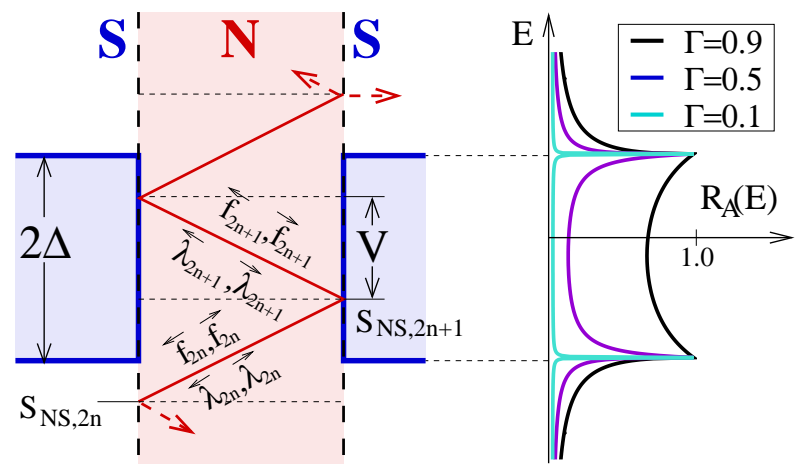

FIG. 1: Left panel: A schematic of the geometry and the MAR-ladder in energy space (see text for details). Right panel: Energy dependent probability $R_{A}(E)$ of a single Andreev reflection for different interface transparencies $\Gamma$. 
in the normal region (for incoherent transport in diffusive NS-systems, see Refs. 20]). We assume low temperature, $k T \ll \Delta$, and negligible relaxation due to electronelectron or electron-phonon scattering. Furthermore, we focus on semiclassical systems with $M \gg 1$.

In this situation, following OTBK 16], the state of the normal region is described by energy, space and direction dependent occupation functions $\overleftarrow{f_{n}}, \overrightarrow{f_{n}}$ for leftand right-going particles ( $n$ even) and holes ( $n$ odd) [see Fig. 回. The average current through the junction is obtained from a set of coupled linear equations, the OBTKequations, for the occupation functions on different rungs of the energy ladder. In the present work, we show how to extend the OBTK-equations to determine the current noise and the FCS in the system. We however emphasize that incoherent diffusive [8, 2, 10] or chaotic contacts [21] can be treated in a very similar fashion. Below we put $e=\hbar=1$.

Introduced by Levitov and Lesovik 11], the FCS

$$
P(Q)=\frac{1}{2 \pi} \int d \chi e^{-i \chi Q} e^{S(i \chi)}, \quad Q=\int_{0}^{\tau} d t I(t),
$$

is the distribution giving the probability that a certain charge $Q$ flows through an electrical conductor during the time interval $[0, \tau]$. It describes entirely the lowfrequency current fluctuations, if $\tau$ is chosen longer than any intrinsic time scale. The Fourier transform of the probability distribution $P(Q)$ yields the generating function $S(\chi)=\sum \chi^{p}\left\langle Q^{p}\right\rangle / p$ ! of irreducible cumulants and gives the mean current $I=\langle Q\rangle / \tau$, the current noise $S_{I}=\left\langle Q^{2}\right\rangle / \tau$, the third cumulant $C_{3}=\left\langle Q^{3}\right\rangle / \tau$ describing the asymmetry of the distribution, etc.

Formal Solution - The FCS of a single NS-interface is a multinomial process and can be fully described 22] by the reflection and transmission probabilities $R_{N / A}, T_{N / A}$. Inside the gap, $|E|<\Delta$, the generating function is

$$
\begin{array}{r}
S_{\mathrm{NS}}=\frac{M \tau}{2 \pi} \ln \left\{f_{p} f_{h}+f_{p}\left(1-f_{h}\right)\left(R_{A} e^{2 \chi}+R_{N}\right)\right. \\
\left.+f_{h}\left(1-f_{p}\right)\left(R_{A} e^{-2 \chi}+R_{N}\right)+\left(1-f_{p}\right)\left(1-f_{h}\right)\right\}
\end{array}
$$

where $f_{p}, f_{h}$ are the particle and hole occupation functions in the normal region. A similar expression accounts for reflection and transmission outside the gap, $|E|>\Delta$. The SNS-junction can be considered as a series of two NS-interfaces sharing the same occupation functions. In the incoherent semiclassical limit, the FCS of such a compound system can be calculated with the stochastic path integral formalism [14, 15]. The key ingredient in this formalism is a separation of time scales for fast collision events (the Andreev reflections) and for the slow evolution of the occupation functions. This separation allows one to integrate out the fast fluctuations and to express the generating function $S(\chi)$ by an action in terms of slow collective variables as,

$$
S[\chi, f, \lambda]=\int_{0}^{2 V} d E \sum_{n=-\infty}^{\infty} S_{\mathrm{NS}, n}\left(E_{n}\right),
$$

where the integral is over the different ladders, contributing independently to the action. The actions $S_{\mathrm{NS}, n}$ evaluated at $E_{n}=E+n V$ (see Fig. (1) are generalizations of Eq. (3) which distinguish left- and right-going currents. E.g. for the left interface ( $n$ even), for $\left|E_{n}\right|<\Delta$ we find

$$
\begin{gathered}
S_{\mathrm{NS}, n}\left(E_{n}\right)=\frac{M \tau}{2 \pi} \ln \left\{\overleftarrow{f_{n}-1} \overleftarrow{f_{n}} e^{\overrightarrow{\lambda_{n}}+1}+\overrightarrow{\lambda_{n}}-\overleftarrow{\lambda_{n}-1}-\overleftarrow{\lambda_{n}}+\right. \\
\overleftarrow{f_{n}-1}\left(1-\overleftarrow{f_{n}}\right)\left(R_{A} e^{2 \chi+\overrightarrow{\lambda_{n}}-\overleftarrow{\lambda_{n}}-1}+R_{N} e^{\overrightarrow{\lambda_{n}}-\overleftarrow{\lambda_{n}}-1}\right)+ \\
\overleftarrow{f_{n}}\left(1-\overleftarrow{f_{n}}-1\right)\left(R_{A} e^{-2 \chi+\overrightarrow{\lambda_{n}}-\overleftarrow{\lambda_{n}}}+R_{N} e^{\overrightarrow{\lambda_{n}}-\overleftarrow{\lambda_{n}}}\right) \\
\left.+\left(1-\overleftarrow{f_{n}}-1\right)\left(1-\overleftarrow{f_{n}}\right)\right\}
\end{gathered}
$$

In addition to the occupation functions $\overleftarrow{f_{n}}, \overrightarrow{f_{n}}$ we introduce internal counting fields $\overleftarrow{\lambda_{n}}, \overrightarrow{\lambda_{n}}$ which are Lagrange multipliers preventing charge accumulation inside the normal region. The action (4) has to be varied over all possible configurations of $f_{n}$ 's and $\lambda_{n}$ 's [14]. In the semiclassical regime, we may calculate the action in the saddle point approximation using the equations of motion [14],

$$
\frac{\partial S}{\partial \overleftarrow{\lambda}_{n}}=\frac{\partial S}{\partial \vec{\lambda}_{n}}=\frac{\partial S}{\partial \overleftarrow{f}_{n}}=\frac{\partial S}{\partial \vec{f}_{n}}=0
$$

These equations form an infinite system of coupled nonlinear equations which have to be solved for arbitrary $\chi$ [the occupation of incoming quasiparticles is unity (zero) at $E<-\Delta(E>\Delta)$ ]. The solutions are then substituted back into Eq. (4) to obtain $S(\chi)$. The Fourier transform (2) yielding the probability distribution $P(Q)$ can be carried out in the stationary phase approximation. The relation to the OBTK equations becomes clear if we calculate the average current $I=d S(\chi) /\left.d \chi\right|_{\chi=0} / \tau$. For this purpose, it is sufficient to solve the saddle point equations for $\chi=0$, giving $\overleftarrow{\lambda}_{n}, \vec{\lambda}_{n}=0$. The first two derivatives in Eq. (6) then become exactly the OBTK equations for the occupation functions. For the noise $S_{I}=d^{2} S(\chi) /\left.d \chi^{2}\right|_{\chi=0} / \tau$, this procedure is no longer adequate and the saddle point equations then have to be solved to first order in $\chi$.

Numerical Results - In general, the FCS has to be calculated numerically. A typical distribution function $P(Q)$ is shown in Fig. 2 the FCS is asymmetric, bounded from the left side, $Q>0$ (since charge may not flow against the bias) and exhibits a long tail on the right side (since the overall probability to undergo MAR and to climb up the energy ladder is small). Saddle point solutions for the $f$ 's belonging to different points of the 


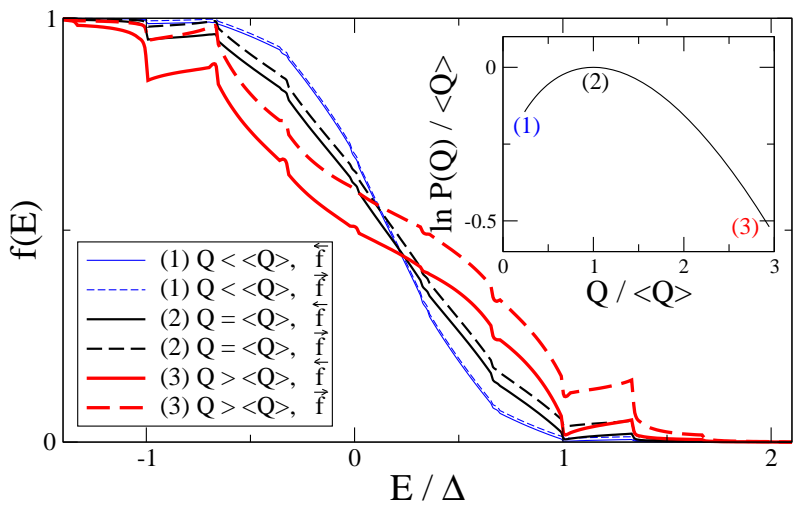

FIG. 2: The FCS for $V=0.34 \Delta$ and $\Gamma=0.5$. Main panel: The left- and rightgoing distribution functions at the right interface for a transmitted charge $Q<\langle Q\rangle, Q=\langle Q\rangle$ and $Q>\langle Q\rangle$. Upper inset: The probability distribution $P(Q)$ of transmitted charge.

probability distribution show characteristic structures at $E= \pm \Delta+n V$ due to the cusps in the Andreev reflection probability (see Fig. 1). The occupation functions $\vec{f}, \overleftarrow{f}$ can be interpreted as the most probable configurations under the condition that a certain charge $Q \propto \int d E(\vec{f}-\overleftarrow{f})$ is transmitted. If the output is the mean charge $Q=\langle Q\rangle$, the solution is exactly the one found by the OBTK equations. For a small amount of transmitted charge $Q<\langle Q\rangle$, the difference $\vec{f}-\overleftarrow{f}$ becomes tiny (no net current). In the opposite limit $Q>$ $\langle Q\rangle$, this difference is large.

The differential FCS, shown in Fig. 3] show a pronounced subharmonic gap structure, with cusps at voltages $V=2 \Delta / n$. Note that the derivative of the probability distribution with respect to voltage is always negative, i.e. the distribution gets broader towards the low voltage limit and acquires a strong tail for $Q>\langle Q\rangle$. The first three differential cumulants $d\left\langle Q^{n}\right\rangle / d V$ are plotted in Fig. 4 for transparencies $\Gamma=0.1,0.5$ and 0.9. All cumulants show a subharmonic gap structure, more pronounced for the noise and third cumulant than for the current 23].

Voltage limits - For high voltages $V \gg \Delta$, only quasiparticles traversing the junction once contribute to the differential transport statistics. The saddle point equations in (6) can then be solved analytically, giving the FCS $[q=Q /(M V \tau /(2 \pi))]$

$\frac{\partial \ln P(Q)}{\partial V}=\frac{M \tau}{2 \pi}\left\{\ln \frac{(1-\Gamma)(1+q)}{1-q}-q \ln \frac{4(1-\Gamma) q^{2}}{1-q^{2}}\right\}$,

the result for the double barrier system in Fig. 1 in the normal state 24]. Unlike the numerical result for intermediate voltages in Fig. 3 this high voltage result scales linearly with $V$ and is bounded from above $(0<q<1)$.

For low voltage $V \ll \Delta$, the quasiparticles injected below the gap traverse the junction a large number of times $N=2 \Delta / V \gg 1$ before being emitted above the

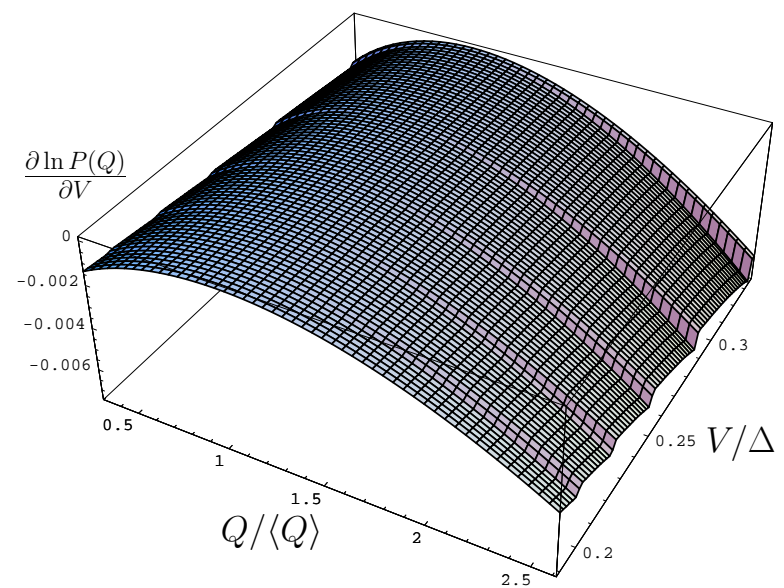

FIG. 3: Differential full counting statistics as function of the voltage for the MAR-system in the tunneling limit, $\Gamma=0.1$.

gap. For sufficiently strong normal backscattering at the NS-interfaces, $\Gamma^{N} \ll \Gamma / N$, the motion in energy space, along the ladder in Fig. $\square$ becomes diffusive [9]. We may introduce a scale $m$ with $1 \ll m \ll N$ below which the motion is ballistic and described by an energy-dependent conductance per rung, $\tilde{G}=M R_{A}(E) /\left(2 \pi\left[1-R_{A}(E)\right]\right)$. On larger scales, one can apply a diffusion approximation $\overleftarrow{f_{n}} \simeq \overrightarrow{f_{n}} \equiv f_{n}$ and $f_{n+m} \simeq f_{n}+m\left(\partial f_{n} / \partial n\right)$ (and similarly for the $\lambda$ 's) to Eq. (5). Replacing the sum in Eq. (4) by an integral (putting $d n=d E / V$ ), we obtain in analogy to the procedure for a normal diffusive wire [15] the action

$$
\frac{S}{2 \tau V^{2}}=\int_{-\Delta}^{\Delta} d E \tilde{G}\left[f(1-f)\left(\frac{\partial \lambda}{\partial E}+\frac{\chi}{V}\right)^{2}-\frac{\partial f}{\partial E}\left(\frac{\partial \lambda}{\partial E}+\frac{\chi}{V}\right)\right]
$$

Changing variables $\lambda \mapsto \lambda+\chi E / V$ and $d E \sim \tilde{G} d y$ maps Eq. (8) onto the action of a normal diffusive wire 15. with renormalized, voltage dependent charge $e \rightarrow e(2 \Delta / V)$. This gives directly the generating function in Eq. (11) with $G=\Delta\left[\int_{-\Delta}^{\Delta} d E \tilde{G}^{-1}\right]^{-1}=3 M \Gamma^{2} /(2 \pi[16(1-\Gamma)])$. Consequently, for low voltages, the current $I=V G$ is proportional to voltage, while the noise $S_{I}=4 \Delta G / 3$ saturates a a constant value. All higher cumulants $p \geq 3$ diverge as $V^{2-p}$.

Generalization - Importantly, the stochastic path integral method presented here can be employed to any semiclassical, incoherent mesoscopic SNS-junction. In particular, Eq. (11) gives the low voltage FCS of any SNSjunction with sufficient normal back-scattering. We emphasize that the FCS is characterized by a single parameter, the low-voltage conductance $G$. For a diffusive normal region with conductance $G_{W}$ we find for instance $G=\left[4 \pi\left(8(1-\Gamma)+3 \Gamma^{2}\right) /\left(3 M \Gamma^{2}\right)+2 / G_{W}\right]^{-1}$. For negligible interface resistance, $M /(2 \pi) \gg G_{W}$, Eq. (11) then extends the shot-noise result of Refs. 9, 10 to the FCS. The opposite limit $M /(2 \pi) \ll G_{W}$ corresponds to an incoherent chaotic cavity. One should however keep in 


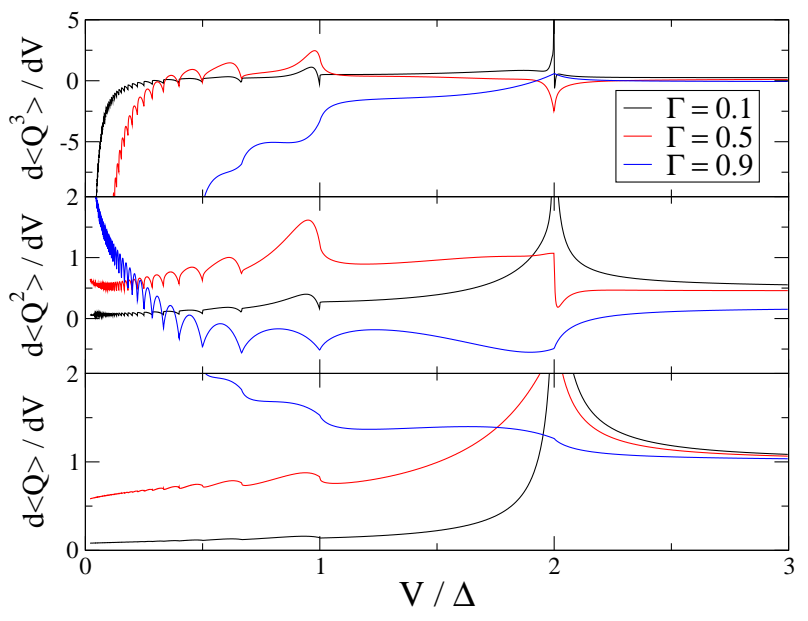

FIG. 4: The first three differential cumulants of the FCS, normalized with the normal state conductance, as a function of voltage for three different transparencies $\Gamma$ of the NSinterfaces.

mind that eventually, for sufficiently low voltage, inelastic electron-electron and electron-phonon scattering will dominate the transport and the low-voltage result in Eq. (1) will not apply.

To access the FCS for the full voltage range, the numerical scheme presented for the OTBK-system has to be appropriately modified. E.g, for the normal region being a chaotic cavity, the only modification is to consider an isotropic occupation function $\overleftarrow{f_{n}}=\overrightarrow{f_{n}}=f_{n}$ (and $\overleftarrow{\lambda_{n}}=\overrightarrow{\lambda_{n}}=\lambda_{n}$ ) in Eq. (5). For a diffusive SNS-junction, one can directly extend the semiclassical approach for current and noise in Refs. [8, 9, 10] to the FCS. At a given voltage $2 \Delta /(n+1)<V<2 \Delta / n$, quasiparticles are transfered through the gap via $n+1$ or $n+2$ traversals across the junction, transporting $n+1$ or $n+2$ charges respectively. The transport is effectively through a diffusive wire of conductance $G_{W} /(n+1)$ or $G_{W} /(n+2)$. The action is then just the sum of the actions of these two diffusive transport processes, with appropriate weights,

$$
\begin{aligned}
& S /\left(G_{W} \tau\right)=\frac{2 \Delta-n V}{n+2} \operatorname{asinh}^{2} \sqrt{\exp [\chi(n+2)]-1} \\
+ & \frac{(n+1) V-2 \Delta}{n+1} \operatorname{asinh}^{2} \sqrt{\exp [\chi(n+1)]-1}
\end{aligned}
$$

The two first cumulants 9, 10] $I=G_{W} V$ and $S_{I}=$ $2 G_{W}(2 \Delta+V) / 3$ are smooth functions of voltage. However, higher cumulants exhibit kinks at $V=2 \Delta / n$.

In conclusion, we have presented a theory for the full counting statistics of incoherent multiple Andreev reflection, based on the stochastic path integral approach. The charge is transfered in quantas of multiple electron charge, giving rise to a low-voltage divergence for cumulants of order three and higher.
This work was supported by the Swiss NSF and the program MaNEP.

[1] T. M. Klapwijk, G. E. Blonder, and M. Tinkham, Physica B+C 109-110, 1657 (1982).

[2] N. Agrait, A. Levy Yeyati, J. M. van Ruitenbeek, Phys. Rep. 377, 81 (2003).

[3] P. Dieleman et al., Phys. Rev. Lett. 79, 3486 (1997).

[4] R. Cron et al., Phys. Rev. Lett. 86, 4104 (2001); F.E. Camino, cond-mat/0406650

[5] X. Jehl et al., Phys. Rev. Lett. 83, 1660 (1999); T. Hoss et al., Phys. Rev. B 62, 4079 (2000); P. Roche et al., Physica C 352, 73 (2001); C. Hoffmann, F. Lefloch, and M. Sanquer, Euro. Phys. J B 29, 629 (2002).

[6] J.P. Hessling et al, Europhys. Lett. 34, 49 (1996); D. Averin and H.T. Imam, Phys. Rev. Lett. 76, 3814 (1996); J.C. Cuevas, A. Martin-Rodero, and A. Levy-Yeyati, ibid 82, 4086 (1999).

[7] Y. Naveh and D.V. Averin, Phys. Rev. Lett. 82, 4090 (1999).

[8] E. V. Bezuglyi et al., Phys. Rev. Lett. 83, 2050 (1999).

[9] K. E. Nagaev, Phys. Rev. Lett. 86, 3112 (2001).

[10] E. V. Bezuglyi et al. Phys. Rev. B 63100501 (2001).

[11] L. S. Levitov and G. B. Lesovik, JETP Lett. 58, 230 (1993); cond-mat/9401004 L. S. Levitov, H. Lee, and G. B. Lesovik, J. Math. Phys. 37, 4845 (1996).

[12] G. Johansson, P. Samuelsson, Å. Ingerman, Phys. Rev. Lett. 91, 187002 (2003).

[13] J. C. Cuevas and W. Belzig, Phys. Rev. Lett. 91, 187001 (2003); condmat/0406508.

[14] S. Pilgram, A. N. Jordan, E. V. Sukhorukov, and M. Büttiker, Phys. Rev. Lett. 90, 206801 (2003).

[15] A. N. Jordan, E. V. Sukhorukov, and S. Pilgram, cond-mat/0401650

[16] M. Octavio et al., Phys. Rev. B 27, 6739 (1983).

[17] H. Lee, L. S. Levitov, A. Yu. Yakovets, Phys. Rev. B 51, 4079 (1995); Yu. V. Nazarov, Ann. Phys. (Leipzig) 8 Spec. Issue, S1-193 (1999); T. Bodineau and B. Derrida, Phys. Rev. Lett. 92, 180601 (2004); D. B. Gutman, A. D. Mirlin, and Y. Gefen, cond-mat/0403436 (2004).

[18] B. Reulet, J. Senzier, and D. E. Prober, Phys. Rev. Lett. 91, 196601 (2003).

[19] G. E. Blonder, M. Tinkham, and T. M. Klapwijk, Phys. Rev. B 27, 112 (1983).

[20] E. V. Bezuglyi et al., Phys. Rev. B 62, 14439 (2000); K. Nagaev and M. Büttiker, ibid 63, 081301 (2001); W. Belzig and P. Samuelsson, Europhys. Lett. 64, 253 (2003).

[21] P. Samuelsson et al, Phys. Rev. B 65, 180514 (2002).

[22] B. A. Muzykantskii and D. E. Khmelnitskii, Phys. Rev. B 50, 3982 (1994); W. Belzig and Yu. V. Nazarov, Phys. Rev. Lett. 87, 197006 (2001).

[23] The semiclassical noise-theory of Ref. [3] gives a qualitatively similar but quantitatively different result.

[24] M. J. M. de Jong, Phys. Rev. B 54, 8144 (1996); P.E. Roche, B. Derrida, and B. Douçot, cond-mat/0312659 